\title{
Can 5-HT 3 Antagonists Really Contribute to Serotonin Toxicity? A Call for Clarity and Pharmacological Law and Order
}

\author{
Carlos H. Rojas-Fernandez
}

Published online: 17 October 2014

(C) The Author(s) 2014. This article is published with open access at Springerlink.com

Since 2012, the World Health Organisation, US Food and Drug Administration, and recently Health Canada have released reports concluding that there is a potential risk of developing serotonin syndrome when $5-\mathrm{HT}_{3}$ antagonists are used in combination with serotonergic drugs. These reports do not appear to have adequately considered current knowledge and understanding of serotonin toxicity (ST), have made conclusions based upon biologically implausible mechanisms, and have not provided health professionals with accurate or useful information. This commentary aims to clarify these misconceptions in order to aid clinical decision making when using $5-\mathrm{HT}_{3}$ antagonists, which are unlikely to cause ST.

\section{Key Points}

Recent reports have suggested that $5-\mathrm{HT}_{3}$ antagonists contribute to serotonin syndrome when used with serotonergic drugs.

Serotonin toxicity is a predictable consequence of excessive synaptic and peripheral serotonin secondary to the use of combinations of drugs that can sufficiently raise serotonin levels.

The 5- $\mathrm{HT}_{3}$ antagonists do not possess the pharmacological properties necessary to contribute to serotonergic effects and are not reliably documented as causing or contributing to ST. To justify the postulate that they are involved in ST will require clear and convincing evidence, which does not presently exist

C. H. Rojas-Fernandez $(\square)$

Schlegel Research Chair in Geriatric Pharmacotherapy Schlegel-

UW Research Institute on Ageing \& School of Pharmacy,

University of Waterloo, Waterloo, Canada

e-mail: crojas-f@uwaterloo.ca
In May 2014, and without substantive evidential foundation, Health Canada (HC) proposed the notion that $5-\mathrm{HT}_{3}$ antagonists contribute to serotonin syndrome [SS, a.k.a. serotonin toxicity (ST)] [1]. The World Health Organisation (WHO) and US Food and Drug Administration (FDA) have recently come to similar conclusions [2, 3]. The analogy that appropriately describes this proposition is "the emperor's new clothes" because there is insufficient scientific evidence to support it. It represents the creation of a myth and falls into the realm of unsupported speculation.

The review by HC was prompted by a WHO report indicating that ondansetron may contribute to the development of ST when used with serotonergic drugs. The WHO report suggested that there may be an increased vulnerability with the concomitant use of $5-\mathrm{HT}_{3}$ antagonists and serotonergic drugs whereby excessive stimulation of other serotonin $(5-\mathrm{HT})$ receptors such as $5-\mathrm{HT}_{1 \mathrm{a}}$ and $5-\mathrm{HT}_{2 \mathrm{a}}$ results from increasing levels of 5-HT due to $5-\mathrm{HT}_{3}$ receptor antagonism [2]. Similarly, $\mathrm{HC}$ concluded with a notice that manufacturers have been requested to incorporate this information in the warnings and precautions section and issued an information update to the public communicating the risk of ST when $5-\mathrm{HT}_{3}$ antagonists are used with serotonergic drugs [1].

The authors of the FDA, WHO, and HC reports do not appear to recognise or assign sufficient weight to the wellestablished data concerning the pharmacology, mechanisms, and characteristics of ST. This is surprising considering the availability of elegant reviews on this topic and the availability of data from the Hunter Area Toxicology Service data set, which have provided ample evidence for the spectrum concept of ST and for a dose-response relationship mediated by the degree of synaptic serotonin elevation [4-7]. 
It is useful to address the misuse of the term "serotonin syndrome". The term syndrome suggests an idiosyncratic reaction, and although it may in part be accurate because of its inherent definition, "...the collection of signs and symptoms that are observed in, and characteristic of, a single condition", it tends to be misleading when referring to pharmacological toxicity versus side effects [4]. Serotonin toxicity predictably results from excessive synaptic and peripheral serotonin due to use of combinations of drugs that can sufficiently raise serotonin levels and is not idiosyncratic. Given its predictability, it is more accurate to refer to this as serotonin toxicity, just as we refer to lithium toxicity when plasma levels are sufficiently elevated versus adverse effects due to lithium, which may be observed even at lower lithium levels. On multiple occasions others have likewise suggested that using the term ST to describe typical side effects of therapeutic doses of drugs is illogical; furthermore, use of ST might be best reserved to describe more severe cases (akin to poisoning versus side effects) [5-7]. As alluded to, toxicity attributable to excess serotonin may be observed along a spectrum ranging from common serotonergic side effects such as nausea, diarrhoea, insomnia, nervousness, tremor, loose stools, mydriasis, and mild anxiety, through to moderate ST with hyperreflexia, diaphoresis, agitation, dysphoria, restlessness, inducible clonus, and temperatures up to $39^{\circ} \mathrm{C}$, and lastly to severe $\mathrm{ST}$ with sustained clonus, rigidity, and temperatures $>39{ }^{\circ} \mathrm{C}[5]$.

Another issue that merits clarification centres around which classes of drugs are serotonergic and thus capable of precipitating severe ST in certain combinations. It is useful to consider these questions: (1) Can the drug lead to serotonergic side effects? (2) Are serotonergic side effects observed in overdose? (3) If co-administered with monoamine oxidase inhibitors (MAOIs), are moderate to severe serotonergic side effects observed? If the answer to any of these questions is yes, then the drug may possess relevant serotonergic properties, namely MAOIs, serotonin reuptake inhibitors (SRIs), and presynaptic serotonin releasers (e.g. MDMA) [4-7]. In cases of overdose or in certain combinations, these drugs can sufficiently elevate synaptic serotonin and cause overt toxicity. Yet it should be noted that altering each of these mechanisms individually will produce only a specific maximum effect, as demonstrated by the observation that overdoses of SRIs alone do not precipitate severe or fatal ST, or temperature elevations above $38.5{ }^{\circ} \mathrm{C}$ [4-7]. Conversely, $25 \%$ of overdoses of SRIs and moclobemide will result in life-threatening ST, the risk being higher with older MAOIs [4]. This makes sense when considering the pharmacology of the drugs and the physiological mechanisms that regulate 5-HT at the neuronal level [4, 8]. SRIs will acutely elevate synaptic 5-HT by inhibiting its reuptake, but good evidence demonstrates that there is a ceiling effect [6, 7]. Once 5-HT reuptake has been maximally inhibited, there is nothing else that can raise 5-HT levels except to inhibit its breakdown vis-à-vis adding a MAOI, making the combination predictably toxic [4]. As for ondansetron, this 5- $\mathrm{HT}_{3}$ antagonist possesses no agonist properties and thus would not be expected to produce serotonergic effects [9].

The references cited in the HC report do not support the mechanism proposed therein. One is a report of two patients who did not have signs or symptoms that are consistent with ST, and one of the cases involved mirtazapine, a drug with no clinically relevant serotonergic properties [4, 6, 10]. The FDA's report cites the same speculative mechanism from Turkel et al., which was uncritically restated by Altman et al. [11]. The conclusions of the reports by $\mathrm{HC}$ and the WHO appear to be a misunderstanding regarding the discussion in a second report of ST because of concomitant use of SRIs and methylene blue (which is a potent MAOI) [12]. Interestingly, almost 20 years ago, Bailey investigated the concomitant use of ondansetron to reduce nausea secondary to fluvoxamine, with no apparent side effects, and two recent reports of ondansetron augmentation of SRI therapy in patients with obsessive compulsive disorder did not reveal serotonergic side effects [13-15].

The speculation that "excess" serotonin would be available to stimulate $5-\mathrm{HT}_{1 \mathrm{a}}$ receptors is also difficult to reconcile given the distribution and function of $5-\mathrm{HT}_{1 \mathrm{a}}$ receptors, observations that this receptor is unlikely to play a major role in $\mathrm{ST}$, and that $5-\mathrm{HT}_{1 \mathrm{a}}$ partial agonists (e.g. buspirone, aripiprazole) have not been shown to contribute to severe ST [5, 7]. In contrast, implication of the $5 \mathrm{HT}_{2 \mathrm{a}}$ receptor is supported by beneficial effects of $5-\mathrm{HT}_{2 \mathrm{a}}$ antagonists in the treatment of ST [4, 7]. Furthermore, the degree of 5-HT elevation necessary for relevant toxicity is 10-50 times the baseline level, which is hardly achievable from this presumed excess 5-HT that is not bound to $5-\mathrm{HT}_{3}$ receptors [6]. It should also be noted that any peripherally released 5-HT would not cross an intact blood-brain barrier and thus could not contribute to CNS serotonergic effects, without which ST could not be precipitated [8].

The communication to the public and health professionals about a proposed risk of ST from use of $5-\mathrm{HT}_{3 \mathrm{a}}$ blockers and serotonergic drugs may have multiple unintended consequences, including, but not limited to: needless avoidance of ondansetron for patients taking SRIs, unnecessary and potentially dangerous situations where SRIs are abruptly discontinued, and health care professionals having to spend extra time explaining this issue to patients. At worst, it may make it difficult for future warnings from $\mathrm{HC}$, the $\mathrm{WHO}$, or the FDA to be taken seriously. Clinicians need to have access to reliable information so that they know what drug combinations must 
always be avoided (e.g. MAOIs and SRIs), what combinations may be used safely (e.g. SRIs and trazodone or mirtazapine), and which drugs can elevate serotonin, and be aware of commonly used drugs which also possess SRI properties (e.g. meperidine, tramadol, fentanyl, pentazocine) or MAOI properties (e.g. linezolid) $[4,5,7]$.

It is well known that case reports are the lowest level of evidence, but this is not to say they are not useful. Indeed, case reports have revealed many important side effects previously unappreciated (e.g. terfenadine and torsade de pointes), but their misinterpretation and misuse can be problematic. The cases from the FDA, WHO, and HC reports have multiple confounders and are not consistent with recognised diagnostic criteria for ST, which greatly diminish their utility. Indeed, in their present form, the quality of these case reports is not sufficient to make any conclusions.

In order to avoid confusion and misdiagnosing ST, clinicians need to become well informed about the concept of ST as a spectrum of toxicity ranging from mild serotonergic effects such as nervousness, insomnia, and nausea to moderate and severe life-threatening ST with hyperthermia, sustained clonus, and severe rigidity. Clinicians also need to know which drugs possess serotonergic properties and are thus capable of contributing to ST. The information is readily available in the literature. Lastly, clinicians need to scientifically and systematically assess the evidence, assign proper weight to evidence in a manner that is commensurate with its quality in a balanced way, and not assign undue weight to evidence of poor quality based on untenable biological mechanisms.

In conclusion, almost all implicated drugs have been reliably and reproducibly assayed with human cloned receptor (and other) methodologies, and these properties have been shown to be reliable predictors of their propensity to induce ST. Thus, certain drugs and drug-drug combinations predictably induce large elevations of serotonin and therefore cause ST. Ondansetron and related compounds have been demonstrated not to possess these properties to a significant degree and are not reliably documented as causing ST. It is therefore highly doubtful that these drugs are capable of contributing to ST, a conclusion that can be confidently predicted from their pharmacological actions. Therefore, to justify the postulate that they are involved in ST will require clear and convincing evidence, which we can contend does not presently exist.

Open Access This article is distributed under the terms of the Creative Commons Attribution Noncommercial License which permits any noncommercial use, distribution, and reproduction in any medium, provided the original author(s) and the source are credited.

\section{References}

1. Summary safety review-serotonin blocking drugs (serotonin antagonists) Aloxi (palonosetron), Anzemet (dolasetron), Kytril (granisetron) and generics, and Zofran (ondansetron) and generics-Serotonin Syndrome. Available at: http://www.hc-sc.gc.ca/ dhp-mps/medeff/advisories-avis/review-examen/serotonin-eng. php. Accessed May 29th 2014.

2. WHO pharmaceutical newsletter: ondansetron and serotonin syndrome. 2012;3:16-21.

3. Kytrtil 5HT3 safety review. Available at: http://www.fda.gov/ downloads/AdvisoryCommittees/CommitteesMeetingMaterials/ PediatricAdvisoryCommittee/UCM342225.pdf. Accessed 29 May 2014.

4. Gillman PK. A review of serotonin toxicity data: implications for the mechanisms of antidepressant drug action. Biol Psych. 2006;59:1046-51.

5. Gillman PK. CNS toxicity involving methylene blue: the exemplar for understanding and predicting drug interactions that precipitate serotonin toxicity. J Psychopharmacol. 2011;25:429-36.

6. Dunkley EJC, Isbister GK, Sibbritt D, et al. The hunter serotonin toxicity criteria: simple and accurate diagnostic decision rules for serotonin toxicity. Q J Med. 2003;96:634-42.

7. Isbister GK, Buckley NA. The pathophysiology of serotonin toxicity in animals and humans. Implications for diagnosis and treatment. Clin Neuropharmacol. 2005;28:205-14.

8. Frazer A, Hensler JG. Serotonin. In: Siegel GJ, Agranoff BW, Albers RW, Fisher SK, Uhler MD, editors. Basic neurochemistry: molecular, cellular, and medical aspects. 6th ed. Philadelphia: Lippincott-Raven; 1999. p. 264-92.

9. Gregory RE, Ettinger DS. 5- $-\mathrm{HT}_{3}$ receptor antagonists for the prevention of chemotherapy-induced nausea and vomiting. A comparison of their pharmacology and clinical efficacy. Drugs. 1998;55:173-89.

10. Turkel SB, Nadala JGB, Wincor MZ. Possible serotonin syndrome in association with $5-\mathrm{HT}_{3}$ antagonist agents. Psychosomatics. 2001;42:258-60.

11. Altman CS, Jahangiri MF. Serotonin syndrome in the perioperative period. Anesth Analg. 2010;110:526-8.

12. Stanford SC, Stanford BJ, Gillman PK. Risk of severe serotonin toxicity following co-administration of methylene blue and serotonin reuptake inhibitors: an update on a case report of postoperative delirium. J Psychopharmacol. 2010;24:1433-8.

13. Bailey JE, Potokar J, Coupland $\mathrm{N}$, et al. The $5-\mathrm{HT}_{3}$ antagonist ondansetron reduces gastrointestinal side effects induced by a specific serotonin re-uptake inhibitor in man. J Psychopharmacol. 1995;9:137-41.

14. Pallanti S, Bernardi S, Antonini S, et al. Ondansetron augmentation in treatment resistant obsessive compulsive disorder. A preliminary, single blind, prospective study. CNS Drugs. 2009;23:1047-55.

15. Soltani F, Sayyah M, Feizy F, et al. A double blind, placebocontrolled pilot study of ondansetron for patients with obsessive compulsive disorder. Hum Psychopharmacol Clin Exp. 2010;25:509-13. 